\title{
The quality of aphids as food for generalist predators: implications for natural control of aphids
}

\author{
SøREN TOFT \\ Department of Ecology \& Genetics, Institute of Biological Sciences, Aarhus University, Building 540, DK-8000 Århus C, Denmark; \\ e-mail: soeren.toft@biology.au.dk
}

Key words. Polyphagous aphidiphages, specialist aphidiphages, true generalist predators, alternative prey, Araneae, Opiliones, Coleoptera, Hymenoptera, Dictyoptera, Aves

\begin{abstract}
The paper reviews existing data on the food quality of cereal aphids for generalist predators. Data are presented for spiders, harvestmen, carabid and staphylinid beetles, cockroaches, ants and one species of bird. All results agree that cereal aphids are low-quality food compared to alternative prey types (in most studies fruit flies). This is associated both with a low consumption capacity for aphids and a low utilization efficiency of the aphid food. A pure aphid diet allows full juvenile development in only a few species. Aphids as part of mixed diets can have negative, neutral or positive effects, which depends on the quality of the remaining diet. The low consumption capacity for aphids is due to the development of a specific feeding aversion. Genetic variation in the ability to tolerate aphids has been documented, indicating that predators may be able to adapt to a higher proportion of aphids in the diet in areas where outbreaks are frequent. A consequence of these findings is that predator populations rely on alternative prey (e.g. Collembola and Diptera) for maintenance and reproduction, and are probably unable to benefit nutritionally from an aphid outbreak. The low food quality of aphids to generalist predators explains why generalist and specialist predators have widely different roles in aphid biocontrol, but does not rule out that under some conditions the generalists may be able to inhibit aphid population growth sufficiently to prevent an outbreak, as field experiments have indicated. Simulation modelling shows that a low consumption capacity for aphids has little influence on the ability to prevent aphid population increase at low aphid immigration rates, but a great influence at high aphid immigration rates. Modelling also indicates that there may be an optimal availability of high-quality alternative prey that maximizes the impact of generalist predators on aphid population growth.
\end{abstract}

\section{INTRODUCTION}

The natural control of aphids in cereal fields can be viewed as the result of a combined action of generalist predators and more or less specialized aphidophagous predators. Because the generalists can subsist and reproduce on prey other than the aphids, they are present in the fields and may exert their influence at the time the aphids first arrive there, i.e. before the aphids have multiplied to create an outbreak. Under certain conditions they may inhibit aphid multiplication to the extent that the aphids do not reach outbreak levels. Specialist aphid predators on the other hand, depend on aphids for reproduction. They invade the fields only if the aphid populations have reached a sufficient level to induce egg laying. In this situation they may multiply quickly and sometimes be able to graze down an existing aphid outbreak. The two predator groups thus play very different roles in aphid control and exert their effect in different phases of the aphid population cycle: the generalists may work as a preventive force against a prospective outbreak, whereas the specialists may suppress an ongoing outbreak.

As regards the role of generalist predators, the above scenario is based on field experimental work pioneered by Edwards et al. (1979), and followed up by several subsequent studies (reviewed by Symondson et al., 2002). Most of this work used barriers to exclude surface active, non-flying animals; therefore the effects were ascribed mainly to ground beetles, secondarily also to spiders, staphylinid beetles and other taxa. By preventing predator access to experimental plots, predation on immigrating alates or their immediate offspring was hampered, thus allowing the exponential increase of the aphid population. For reasons that are usually unknown, similar aphid outbreaks occur regularly under conventional conditions of cultivation, and generalist predators can do little to combat them. Quantitative studies comparing the impact of generalist versus specialist predators find that aphid specialist predators (aphidophages) then take the larger toll. Thus, Winder et al. (1994) found that syrphid fly larvae were responsible for the largest fraction of aphids eaten, and Schmidt et al. (2003) found that aphid parasitoids were the most effective group for aphid control.

Chang \& Kareiva (1999) identified the generalists' low efficiency as "pest killers" compared to specialist predators as one of the central explanations for their different roles in pest biocontrol. However, little effort seems to have been devoted to identifying the underlying biological reasons, and therefore no satisfactory explanation for the "division of labour" between generalist and specialist predators has been given. Though it is clear why generalists can be present in fields early in the season and specialists cannot, it has been unclear why the generalist predators are so ineffective later in the season when aphid populations are high. As polyphagous feeders the generalists were expected to take advantage of the rich prey availability of an aphid outbreak, but this seems not to be the case. This paper will review results obtained during the last 10 years mainly on the relationships between gen- 
eralist predators and aphid species in an attempt to answer the questions: Why are generalist predators such imperfect aphid controllers, and why do generalist and specialist predators have such different roles in aphid biocontrol? I will argue that we may find at least part of the answer in fundamental differences in the quality of aphids as food for generalist predators and the specialist aphidophages. The review will focus in particular on relationships between aphids and generalist predators of cereal crops in Europe because few comparable data are available from other systems.

\section{A word on terminology}

The literature on aphidophagous predators frequently use the term "polyphagous" for coccinellids, syrphids, chrysopids and others, which include a variety of prey other than aphids in their diets. However, these animals are not as polyphagous in their food choice as are the spiders and carabid beetles that are the focus of the present review. Even the most polyphagous of them show a close association with aphids, which are essential (sensu Hodek \& Honěk, 1996) prey for them, while most non-aphid prey are secondary prey, the latter term meaning that they often do not sustain growth and reproduction, but may improve survival in a starving situation (e.g. Evans \& Gunther, 2005). As will be described below, the situation is basically opposite in spiders and carabid beetles. No single prey order is particularly suitable to generalist predators; both high-quality and low-quality prey can be found within the same orders (e.g. Collembola and Diptera; Toft \& Wise, 1999a). These I will therefore refer to as "true generalist predators" to distinguish them from the more or less aphidophagous species, which will be named "polyphagous aphidophages" or "specialist aphidophages" depending on their reliance on aphids. They include coccinellid beetles, syrphid flies, chrysopid lacewings, certain heteropteran bugs. The generalist predators I will not consider as truly aphidophagous for reasons that will be clear at the end of this review.

"Alternative prey" is used in the literature on aphidophages for low-quality prey that may sustain survival but do not permit growth and reproduction (Hodek \& Honěk, 1996). However, in the literature on biocontrol by generalist predators it is used to describe (high-quality) prey other than the focal pest, i.e. the prey that sustains the predator populations. The term will here be used with the latter meaning.

\section{NUTRITIONAL ECOLOGY OF APHID PREDATION}

The central nutritional ecological question in the context of aphid predation is: to what extent can a predator satisfy its food demand by eating aphids? This question has two aspects: (1) the quantitative aspect: can the predator get satiated by eating aphids? i.e. is the consumption capacity for aphids the same as its total feeding capacity? and (2) the qualitative aspect: does aphid consumption fulfil the nutritional requirements of the predator?

For the aphidophagous predators, the answer is simple: they can, by definition, satisfy their food demand both quantitatively and qualitatively by eating aphids. This is true for both the specialist and polyphagous aphidophages, but not for the true generalist predators.

\section{Consumption capacity for aphids}

Many generalist predators show a lower consumption capacity for aphids compared to other prey types. For some species the aphid consumption capacity is far below that of other prey, for others the difference is small. Table 1 summarises information from studies that specifically made this comparison, including data from two orders of arachnids and two orders of insects. All the generalist predators studied have aphid consumption capacities below their total food demand, defined in most cases by their consumption of laboratory reared fruit flies (Drosophila melanogaster). Even the extremely omnivorous cockroach Blatella germanica will eat a lower mass of aphids than of fruit flies (Nørgaard, 1998). Only from two major taxa, the spiders and the ground beetles, have several species been investigated, allowing a broader comparison. It is clear that spiders generally show a quite low tolerance ( $\sim$ relative consumption capacity) to aphids, while that of carabid beetles generally is much higher. Thus, among spiders the consumption of aphids (Rhopalosiphum padi) amounted to maximally $25 \%$ of fruit fly consumption. Among carabid beetles less than 50\% lower aphid consumption capacity is widespread, but only a few species are able to consume aphids in amounts similar to fruit flies. Also vertebrate insectivores have reduced capacity for aphid consumption; partridge chicks (Perdix perdix) reduced their total consumption by $33 \%$ during their first five days of life, when the food had a $45 \%$ aphid ( $R$. padi) content compared to a diet of pure chopped grashoppers (Borg \& Toft, 2000). One study has compared the preference of a generalist predator for an aphid $R$. padi derived from the primary and secondary plant hosts, respectively. The wolf spider preferred the winter host morph (Toft, 1996). Data for consumption of other aphid species are very scant; Aphis fabae, however, seems to be even less palatable than R. padi (Toft, 1995).

An interesting aspect seen in both spiders (Toft, 1995) and carabid beetles (Bilde \& Toft, 1994, 1997a, b) is that the aphid consumption capacity is not only low, it is also independent of predator hunger state. Even up to 14 days of starvation did not increase aphid consumption in a wolf spider compared to that of satiated spiders (Toft, 1995). As a result, the discrepancy between aphid consumption and food demand increases as the spiders' hunger level increases. This was true whether aphids were served as a pure diet or as part of a mixed diet that included higher-quality prey. The carabid beetle Agonum dorsale consumed less $R$. padi when the aphid was part of a mixed diet than when given as a pure diet, and this was true both for satiated and starved beetles (Bilde \& Toft, 1994).

The independence of aphid consumption from starvation may indicate the presence of toxins or deterrents in the aphids (i.e. a chemical defence), that set a limit to the amounts that the predator can tolerate. In support of deterrence, fruit flies coated with aphid homogenate 
TABLE 1. Studies of consumption capacity for aphids relative to other prey types in generalist predators. Based on mg dry weight consumed, unless otherwise stated.

\begin{tabular}{|c|c|c|c|c|}
\hline Predator species & Aphid species & Alternative prey species & Relative aphid consumption & Reference \\
\hline \multicolumn{5}{|l|}{ ARANEAE } \\
\hline \multicolumn{5}{|l|}{ Clubionidae } \\
\hline \multirow[t]{6}{*}{ Clubiona sp. (subadults) } & R. padi & D. melanogaster & 우 20\% (satiated) & Madsen et al., 2004 \\
\hline & & & $16 \%(7-d$ starved $)$ & \\
\hline & & & $10 \%(14-d$ starved $)$ & \\
\hline & & & o $11 \%$ (satiated) & \\
\hline & & & $14 \%(7-d$ starved $)$ & \\
\hline & & & $17 \%$ (14-d starved) & \\
\hline \multicolumn{5}{|l|}{ Lycosidae } \\
\hline \multirow[t]{3}{*}{ Pardosa amentata juv. } & R. padi & D. melanogaster & $18 \%$ & Toft, 1995 \\
\hline & R. padi & Bradycera (Sciaridae) & $23 \%$ & \\
\hline & A. fabae & R. padi & $36 \%$ & \\
\hline \multirow[t]{4}{*}{ Pardosa prativaga juv. } & R. padi & D. melanogaster & $10 \%$ (satiated) & Toft, 1995 \\
\hline & & & $5.0 \%(3-d$ starved $)$ & \\
\hline & & & $1.6 \%$ (7-d starved $)$ & \\
\hline & & & $2.4 \%(14-\mathrm{d}$ starved $)$ & \\
\hline \multicolumn{5}{|l|}{ Linyphiidae } \\
\hline \multirow[t]{2}{*}{ Erigone atra } & R. padi & D. melanogaster & $16 \%$ (satiated) & Bilde \& Toft, 1997a \\
\hline & & & $5 \%(7-d$ starved $)$ & \\
\hline Oedothorax apicatus & R. padi & D. melanogaster & $25 \%$ (satiated) & Bilde \& Toft, 1997a \\
\hline & & & $10 \%$ (7-d starved) & \\
\hline Tetragnathidae & & & & \\
\hline Pachygnatha degeeri & R. padi & D. melanogaster & 우 24\% (satiated) & Madsen et al., 2004 \\
\hline & & & $10 \%$ (7-d starved $)$ & \\
\hline & & & $19 \%$ (14-d starved) & \\
\hline & & & o $17 \%$ (satiated) & \\
\hline & & & $15 \%$ (7-d starved) & \\
\hline & & & $22 \%$ (14-d starved) & \\
\hline OPILIONES & & & & \\
\hline Nemastomatidae & & & & \\
\hline Nemastoma lugubre females and males & S. avenae & Sinella curvirostra (Collembola) & $93 \%$ & $\begin{array}{l}\text { A. Hvam \& S. Toft, } \\
\text { unpubl. }\end{array}$ \\
\hline Phalangiidae & & & & \\
\hline Oligolophus tridens females & S. avenae & D. melanogaster & $36 \%$ & \\
\hline Rilaena triangularis juveniles & S. avenae & D. melanogaster & $18 \%$ & \\
\hline Rilaena triangularis females & S. avenae & D. melanogaster & $53 \%$ & \\
\hline COLEOPTERA & & & & \\
\hline Carabidae & & & & \\
\hline Agonum dorsale & R. padi & D. melanogaster & $21 \%$ (satiated) & Bilde \& Toft, 1994 \\
\hline & & & $2 \%$ (sat. - mixed diet) & \\
\hline & & & $10 \%(7-d$ starved $)$ & \\
\hline & & & $6 \%(7-d$ st. - mixed diet $)$ & \\
\hline Agonum dorsale & R. padi & D. melanogaster & $22 \%$ (satiated) & Bilde \& Toft, 1997a \\
\hline & & & $74 \%$ (7-d starved) & \\
\hline Bembidion lampros & R. padi & D. melanogaster & $81 \%$ (satiated) & Sørensen, 1996 \\
\hline & & & $51 \%(7-d$ starved $)$ & \\
\hline & S. avenae & & $72 \%$ (satiated) & \\
\hline & & & $63 \%$ (7-d starved $)$ & \\
\hline & M. dirhodum & & $82 \%$ (satiated) & \\
\hline & & & $60 \%(7-d$ starved $)$ & \\
\hline Calathus fuscipes & R. padi & D. melanogaster & $66 \%$ (satiated) & Bilde \& Toft, 1997a \\
\hline & & & $52 \%(7-d$ starved $)$ & \\
\hline Calathus melanocephalus & R. padi & D. melanogaster & $98 \%$ (satiated) & Bilde \& Toft, 1997a \\
\hline & & & $60 \%$ (7-d starved $)$ & \\
\hline Calathus melanocephalus & R. padi & D. melanogaster & $76 \%$ (satiated) & Bilde \& Toft, 1999 \\
\hline & & & $55 \%$ (7-d starved $)$ & \\
\hline & S. avenae & & $88 \%$ (satiated) & \\
\hline & & & $63 \%$ (7-d starved $)$ & \\
\hline & M. dirhodum & & $72 \%$ (satiated) & \\
\hline & & & $66 \%(7-d$ starved $)$ & \\
\hline Pterostichus melanocephalus & R. padi & D. melanogaster & $57 \%$ (satiated) & Bilde \& Toft, 1997a \\
\hline & & & $53 \%(7-d$ starved $)$ & \\
\hline Staphylinidae & & & & \\
\hline Tachyporus hypnorum Ad. & R. padi & D. melanogaster & $98 \%$ & Sørensen, 1996 \\
\hline & S. avenae & & $74 \%$ & \\
\hline & M. dirhodum & & $45 \%$ & \\
\hline Larvae L1 & R. padi & & $128 \%$ & \\
\hline & S. avenae & & $140 \%$ & \\
\hline & M. dirhodum & & $100 \%$ & \\
\hline Larvae L2 & R. padi & & $48 \%$ & \\
\hline & S. avenae & & $60 \%$ & \\
\hline & M. dirhodum & & $53 \%$ & \\
\hline Larvae L3 & R. padi & & $67 \%$ & \\
\hline & S. avenae & & $79 \%$ & \\
\hline & M. dirhodum & & $59 \%$ & \\
\hline DICTYOPTERA & & & & \\
\hline Blatella germanica & R. padi & D. melanogaster & $68 \%$ & Nørgaard, 1998 \\
\hline
\end{tabular}


showed significantly reduced palatability to $A$. dorsale (Bilde \& Toft, 1994).

\section{Food value of aphids to generalist predators}

The degree to which a predator is adapted to utilize aphids as food may be most easily revealed by recording predator performance when the aphid is presented as the only food in a monotypic diet. However, even if a predator is not well adapted as an aphid consumer, it may nevertheless benefit from accepting aphids as prey. A generalist predator with an extended predatory life will consume many different prey types during its life time, and mixed diets are probably the rule also when relatively short time periods are considered. For evaluating the food quality of aphids, it is therefore useful to distinguish their value as monotypic diets, and their contribution to the quality of a mixed diet (Tables 2 and 3).

\section{Monotypic aphid diets}

Given as monotypic diets, aphids are invariably of low food quality to spiders and adult carabid beetles, and available data on harvestmen, staphylinine beetles, ants and partridge chicks indicate that problems utilizing aphids are widespread among generalist predators (Table 2). Compared with prey of higher quality, cereal aphids as the only food reduce all measured fitness parameters, e.g. female fecundity, egg hatching success, juvenile survival, growth and developmental rate. In several species some parameters showed non-significant changes, but no case of a significantly higher fitness parameter than recorded for an alternative high-quality prey has ever been reported.

A commonly observed effect of a pure aphid diet is no or very little growth and development, with some variation among three cereal aphid species. Studies on wolf spiders (Pardosa prativaga) indicate a quality ranking of Metopolophium dirhodum $>$ Sitobion avenae $>$ R. padi with respect to most fitness parameters examined. Thus, none of the hatchlings fed $R$. padi moulted to the second instar, while $8 \%$ fed $S$. avenae and $36 \%$ fed M. dirhodum did so (Toft, 2000). However, all three aphid species were poor quality food as no spiderling developed to the third instar. The indicated ranking of the three aphid species is repeated in some other generalist predator species, but not all (see below).

Aphids may not be completely useless as food even for those predators that cannot utilize them for development or reproduction. A pure aphid diet improved survivorship in linyphiid and lycosid spiders compared to a starvation treatment (Toft, 1995). Thus, in a situation of severe food limitation aphid consumption may postpone the death of starvation considerably.

If, as hypothesized above, the aphids contain toxins or deterrents as a predator avoidance investment, we expect that mature aphids should invest much in chemical defence because that will secure them a long reproductive life, whereas the small nymphs are expected to give priority to a high growth rate and invest relatively less in chemical defence. By this argument juvenile aphids were hypothesized to be of higher food quality than adult aphids. These predictions were tested and confirmed by Toft (2000). Hatchlings of $P$. prativaga survived longer when fed juvenile than adult $R$. padi, and they had obtained a higher weight at the age of 7 days. Though these differences were significant, they were quite small: none of the spiders moulted to the second instar. Thus, juvenile aphids did not support growth and development any better than adult aphids. However, in a food limited situation selection of juvenile aphids by a generalist predator may prevent death from starvation for a longer time than consumption of adult aphids.

\section{Mixed diets with aphids}

The potential contribution of aphids to the fitness effects of mixed diets for generalist predators is variable (positive, neutral, negative), depending on the aphid species, the predator species, and which fitness parameter is being measured (Table 3 ).

In the linyphiid spider Erigone atra a mixed aphid-fruit fly diet improved female reproductive success significantly compared to a pure fruit fly diet (Toft, 1995). Increases were seen both in the number of egg sacs produced and in the number of eggs per egg sac. On top of this a considerable improvement in the hatching success of the eggs occurred, resulting in a near doubling of the number of young per female. Other positive effects of aphids in mixed diets include increased fecundity of the ground beetle Agonum dorsale (Bilde \& Toft, 1994), and in the food utilization and growth of partridge chicks (Borg \& Toft, 1999, 2000).

Significant negative (i.e. toxic) effects of aphids on fitness parameters of generalist predators are few. Bilde \& Toft (2001) found strong negative effects of adding $S$. avenae to a fruit fly diet on reproductive parameters of $E$. atra, but only small and non-significant reductions when adding $R$. padi and $M$. dirhodum. The quality ranking of the aphid species by $E$. atra thus was: $M$. dirhodum $>R$. padi $>$ S. avenae.

\section{Relation between aphid tolerance and ability to utilize aphids}

Some carabid beetles show a high consumption capacity for aphids (Table 1); for example, in Calathus melanocephalus the ad libitum intake of aphids of satiated individuals is equal that of fruit flies. It is pertinent to ask, if such a high consumption capacity is linked to a better ability to utilise aphids than in species with a lower aphid consumption capacity. But this is not the case: when transferred to aphid diets, female fecundity drops drastically, while it can be maintained at a high level on a fruit fly diet (Bilde \& Toft, 1999). Though the species may show a high aphid consumption in the laboratory, it is doubtful whether there are any fitness benefits in the field of this capacity. Because the predators do not profit from eating aphids, we cannot expect them to aggregate where aphids abound (see further below) and we may have little biocontrol benefit from them in spite of the high aphid consumption capacity. 
TABLE 2. Studies on the quality of aphids as food for generalist predators: monotypic (including mixed-aphid) diets.

\begin{tabular}{|c|c|c|c|c|c|}
\hline Predator species & Aphid species & Alternative prey & Parameter & Aphid-alt.prey comparison & Reference \\
\hline \multicolumn{6}{|l|}{$\begin{array}{l}\text { ARANEAE } \\
\text { Linyphiidae }\end{array}$} \\
\hline \multirow[t]{5}{*}{ Erigone atra } & R.padi & Starvation & $\begin{array}{l}\text { Hatchling survival } \\
\text { Development }\end{array}$ & $\begin{array}{l}\text { Improved } \\
\text { None moulted }\end{array}$ & Toft, 1995 \\
\hline & R. padi & D. melanogaster & $\begin{array}{l}\text { No. eggsacs/female } \\
\text { No. eggs/female } \\
\text { No. young/female }\end{array}$ & $\begin{array}{l}66 \% \text { reduction } \\
82 \% \text { reduction } \\
51 \% \text { reduction }\end{array}$ & Toft, 1995, 1996 \\
\hline & $\begin{array}{l}\text { R. padi } \\
\text { S. avenae } \\
\text { M. dirhodum } \\
R p+S a+M d\end{array}$ & D. melanogaster & No. eggsacs/female & $\begin{array}{l}69 \% \text { reduction } \\
69 \% \text { reduction } \\
72 \% \text { reduction } \\
70 \% \text { reduction }\end{array}$ & Bilde \& Toft, 2001 \\
\hline & $\begin{array}{l}\text { R. padi } \\
\text { S. avenae } \\
\text { M. dirhodum } \\
R p+S a+M d\end{array}$ & D. melanogaster & No. eggs/female & $\begin{array}{l}75 \% \text { reduction } \\
83 \% \text { reduction } \\
86 \% \text { reduction } \\
86 \% \text { reduction }\end{array}$ & \\
\hline & $\begin{array}{l}\text { R. padi } \\
\text { S. avenae } \\
\text { M. dirhodum } \\
R p+S a+M d\end{array}$ & D. melanogaster & No. young/female & $\begin{array}{l}67 \% \text { reduction } \\
69 \% \text { reduction } \\
75 \% \text { reduction } \\
77 \% \text { reduction }\end{array}$ & \\
\hline \multirow[t]{3}{*}{ Tenuiphantes tenuis } & S. avenae & $\begin{array}{l}\text { [Several low-quality } \\
\text { diets] }\end{array}$ & $\begin{array}{l}\text { Hatchling survival } \\
\text { Development }\end{array}$ & $\begin{array}{l}\text { Low } \\
2 \% \text { moulted }\end{array}$ & Sunderland et al., 1996 \\
\hline & R. padi & Mixed collembola & Duration of first instar & $\begin{array}{l}\text { o } 114 \% \text { increase } \\
\text { ot } 236 \% \text { increase }\end{array}$ & Beck \& Toft, 2000 \\
\hline & R. padi & Mixed collembola & Survival of $1^{\text {st }}$ instar & $65 \%$ decrease & \\
\hline \multicolumn{6}{|l|}{ Lycosidae } \\
\hline Pardosa amentata & R. padi & [D. melanogaster $]$ & $\begin{array}{l}\text { Hatchling survival } \\
\text { Development }\end{array}$ & $\begin{array}{l}\text { Low } \\
\text { None moulted }\end{array}$ & Toft, 1995 \\
\hline Pardosa lugubris & R. padi & $\begin{array}{l}{[D . \text { melanogaster and }} \\
\text { Heteromurus nitidus } \\
\text { (Collembola)] }\end{array}$ & $\begin{array}{l}\text { Hatchling survival } \\
\text { Growth } \\
\text { Development }\end{array}$ & $\begin{array}{l}\text { Low } \\
\text { Very low } \\
\text { None moulted }\end{array}$ & $\begin{array}{l}\text { Oelbermann \& Scheu, } \\
2002\end{array}$ \\
\hline \multirow[t]{4}{*}{ Pardosa prativaga } & $\begin{array}{l}\text { R. padi } \\
\text { S. avenae } \\
\text { M. dirhodum } \\
R p+S a+M d\end{array}$ & D. melanogaster & Respiration rate & $\begin{array}{l}88 \% \text { increase } \\
29 \% \text { increase } \\
141 \% \text { increase } \\
112 \% \text { increase }\end{array}$ & Toft \& Nielsen, 1997 \\
\hline & $\begin{array}{l}\text { R. padi } \\
\text { S. avenae } \\
\text { M. dirhodum } \\
R p+S a+M d\end{array}$ & $\begin{array}{l}\text { [D. melanogaster and } \\
\text { starvation] }\end{array}$ & Development & $\begin{array}{l}\text { None moulted } \\
8 \% \text { moulted } \\
36 \% \text { moulted } \\
12 \% \text { moulted }\end{array}$ & Toft, 2000 \\
\hline & $\begin{array}{l}\text { R. padi } \\
\text { S. avenae } \\
\text { M. dirhodum } \\
R p+S a+M d\end{array}$ & Starvation & Survival & $\begin{array}{l}\text { Same as starvation } \\
\text { Improved } \\
\text { Improved } \\
\text { Slightly improved }\end{array}$ & \\
\hline & $\begin{array}{l}\text { R. padi } \\
\text { S. avenae } \\
\text { M. dirhodum } \\
R p+S a+M d\end{array}$ & Starvation & Growth & $\begin{array}{l}\text { Same as starvation } \\
\text { Slightly improved } \\
\text { Slightly improved } \\
\text { Slightly improved }\end{array}$ & \\
\hline Schizocosa sp. & Aphis nerii & [Several] & $\begin{array}{l}\text { Hatchling survival } \\
\text { Development }\end{array}$ & $\begin{array}{l}\text { Low: same as starvation } \\
\text { None moulted }\end{array}$ & Toft \& Wise, 1999a \\
\hline \multicolumn{6}{|l|}{ OPILIONES } \\
\hline \multirow[t]{2}{*}{ Oligolophus tridens } & R. padi & $\begin{array}{l}\text { D. melanogaster and } \\
\text { others }\end{array}$ & $\begin{array}{l}\text { Survival } \\
\text { Development }\end{array}$ & $\begin{array}{l}\text { Low } \\
0-2 \text { moults (out of } 6 \text { ) }\end{array}$ & Hvam \& Toft, in press \\
\hline & S. avenae & $\begin{array}{l}\text { D. melanogaster and } \\
\text { others }\end{array}$ & $\begin{array}{l}\text { Survival } \\
\text { Development }\end{array}$ & $\begin{array}{l}\text { Low } \\
0-2 \text { moults (out of } 6 \text { ) }\end{array}$ & \\
\hline $\begin{array}{l}\text { Rilaena triangularis } \\
\text { females }\end{array}$ & S. avenae & D. melanogaster & Weight change & Negative (pos. in D.m.) & $\begin{array}{l}\text { A. Hvam \& S. Toft, } \\
\text { unpubl. }\end{array}$ \\
\hline \multicolumn{6}{|l|}{$\begin{array}{l}\text { COLEOPTERA } \\
\text { Carabidae }\end{array}$} \\
\hline Agonum dorsale & R. padi & D. melanogaster & Fecundity & $66 \%$ reduction & Bilde \& Toft, 1994 \\
\hline Amara similata & R. padi & Pоа аппиа seeds & $\begin{array}{l}\text { Fecundity rate } \\
\text { (No. eggs/day) }\end{array}$ & $73 \%$ reduction & Jørgensen \& Toft, 1997a \\
\hline & R. padi & Mixed insects & Fecundity rate & $83 \%$ reduction & \\
\hline & M. dirhodum & Mixed insects & Fecundity rate & $69 \%$ reduction & \\
\hline Amara similata larvae & $\begin{array}{l}\text { R. padi } \\
\text { S. avenae } \\
\text { M. dirhodum }\end{array}$ & Mixed insects & Survival & $\begin{array}{l}\text { N.s. } \\
\text { N.s. } \\
\text { N.s. }\end{array}$ & \\
\hline Bembidion lampros & R. padi & Cat food & $\begin{array}{l}\text { Egg volume } \\
\text { Fecundity rate }\end{array}$ & $\begin{array}{l}\text { N.s. } \\
\text { N.s. }\end{array}$ & Wallin et al., 1992 \\
\hline
\end{tabular}


TABLE 2 (continued).

\begin{tabular}{|c|c|c|c|c|c|}
\hline Predator species & Aphid species & Alternative prey & Parameter & Aphid-alt.prey comparison & Reference \\
\hline \multirow{2}{*}{\multicolumn{6}{|c|}{$\begin{array}{l}\text { COLEOPTERA } \\
\text { Carabidae }\end{array}$}} \\
\hline & & & & & \\
\hline Calathus melanocephalus & $\begin{array}{l}\text { R. padi } \\
\text { S. avenae } \\
M . \text { dirhodum } \\
R p+S a+M d\end{array}$ & D. melanogaster & Fecundity & $\begin{array}{l}82 \% \text { reduction } \\
87 \% \text { reduction } \\
81 \% \text { reduction } \\
89 \% \text { reduction }\end{array}$ & Bilde \& Toft, 1999 \\
\hline Harpalus rufipes larvae & R. padi & $\begin{array}{l}{[\text { D. melanogaster }} \\
+ \text { Tenebrio larvae }]\end{array}$ & $\begin{array}{l}\text { Survival } \\
\text { Development }\end{array}$ & $\begin{array}{l}\text { Low } \\
\text { None moulted }\end{array}$ & Jørgensen \& Toft, 1997b \\
\hline Nebria brevicollis & M. persicae & Tenebrio larvae & $\begin{array}{l}\text { Fecundity } \\
\text { Egg mortality }\end{array}$ & $\begin{array}{l}94 \% \text { reduction } \\
\text { N.s. }\end{array}$ & Luff, 2005 \\
\hline \multirow[t]{2}{*}{ Poecilus cupreus } & M. persicae & Tenebrio larvae & $\begin{array}{l}\text { Fecundity } \\
\text { Egg mortality }\end{array}$ & $\begin{array}{l}75 \% \text { reduction } \\
\text { N.s. }\end{array}$ & Luff, 2005 \\
\hline & R. padi & Catfood & $\begin{array}{l}\text { Egg volume } \\
\text { Fecundity rate }\end{array}$ & $\begin{array}{l}\text { N.s. } \\
\text { N.s. }\end{array}$ & Wallin et al., 1992 \\
\hline Pterostichus melanarius & R. padi & Catfood & $\begin{array}{l}\text { Egg volume } \\
\text { Fecundity rate }\end{array}$ & $\begin{array}{l}10 \% \text { reduction } \\
\text { N.s. }\end{array}$ & Wallin et al., 1992 \\
\hline \multicolumn{6}{|l|}{ Staphylinidae } \\
\hline \multirow[t]{2}{*}{ Tachyporus hypnorum } & R. padi & D. melanogaster & $\begin{array}{l}\text { Fecundity } \\
\text { Hatching time } \\
\text { Hatching success } \\
\text { No. young/female }\end{array}$ & $\begin{array}{l}\text { N.s. } \\
6 \% \text { increase } \\
27 \% \text { decrease } \\
25 \% \text { decrease }\end{array}$ & Kyneb \& Toft, in prep. \\
\hline & S. avenae & D. melanogaster & $\begin{array}{l}\text { Fecundity } \\
\text { Hatching time } \\
\text { Hatching success } \\
\text { No. young/female }\end{array}$ & $\begin{array}{l}25 \% \text { decrease } \\
\text { N.s. } \\
20 \% \text { decrease } \\
35 \% \text { decrease }\end{array}$ & \\
\hline \multicolumn{6}{|l|}{$\begin{array}{l}\text { HYMENOPTERA } \\
\text { Formicidae }\end{array}$} \\
\hline Lasius niger & $\begin{array}{l}\text { Aphis fabae }+ \\
\text { honey }\end{array}$ & $\begin{array}{l}\text { D. melanogaster }+ \\
\text { honey }\end{array}$ & $\begin{array}{l}\text { No. eggs } \\
\text { No. larvae } \\
\text { No. pupae } \\
\text { No. workers } \\
\text { Larval weight }\end{array}$ & $\begin{array}{l}25 \% \text { reduction } \\
20 \% \text { reduction } \\
\text { c. } 96 \% \text { reduction } \\
\text { c. } 94 \% \text { reduction } \\
44 \% \text { reduction }\end{array}$ & Offenberg, 1999 \\
\hline \multicolumn{6}{|l|}{ AVES } \\
\hline \multirow[t]{3}{*}{ Perdix perdix chicks } & R. padi & $\begin{array}{l}\text { Grasshopper Locusta } \\
\text { migratoria }\end{array}$ & Juvenile growth & $67 \%$ reduction & Borg \& Toft, 1999 \\
\hline & & & $\begin{array}{l}\text { Food utilization (g } \\
\text { growth } / \mathrm{g} \text { food) }\end{array}$ & $39 \%$ reduction & \\
\hline & & & Flight feather length & $15 \%$ reduction & \\
\hline
\end{tabular}

\section{Some generalists can utilize aphids}

Cereal aphids are not as bad for all of the generalist predators as they are for the spiders. For example, the staphylinid Tachyporus hypnorum may complete its development on a monotypic diet of aphids, though with a low survival probability and a reduced teneral weight compared to individuals raised on fruit flies (Kyneb \& Toft, 2004). Female fecundity is significantly reduced on a monotypic diet of $S$. avenae, but not on $R$. padi. Thus, Tachyporus differs from the other generalist predators in getting a substantial benefit out of aphids, though aphids are still not top quality to them. Similar results have been obtained with the carabid beetle Bembibion lampros, whose larvae complete development on a pure aphid diet, but survival is relatively low (Sørensen, 1996).

\section{The value of aphids in mixed diets depends on the nutritional quality of the remaining diet}

Aphids may sometimes add to, sometimes subtract from the value of mixed diets, as explained above. One way to explain these differences may be that the positive effects are due to aphids containing some nutrients that fruit flies are normally short of, while the negative effects are due to toxins in the aphids. Based on this hypothesis, we pre- dicted that aphids would be a positive addition to a simple nutrient-insufficient diet, but a negative addition to an already nutrient-rich diet. We tested this in a simple experiment (Bilde \& Toft, 2000) by comparing the fitness effects on the linyphiid spider Dicymbium brevisetosum of two mixed diets of fruit flies and R. padi; in one diet the fruit flies were of high nutritional quality, in the other they were of low nutritional quality. Low-quality flies were raised on plain Carolina Drosophila medium, while nutrient-enriched flies were created by adding dog food to the fruit fly medium (Mayntz \& Toft, 2001).

Part of the hypothesis was confirmed by the finding, that adding $R$. padi to enriched flies gave a significantly reduced number of young, while it had no effect to add the same aphid to non-enriched fruit flies. Another part of the hypothesis was confirmed by testing the effects of the maternal diet on the survival of the young, which were all given the same monotypic $R$. padi diet. Survival was significantly improved when aphids were added to nonenriched fruit flies of the maternal diet, but not when added to enriched flies.

In the field predators are likely to encounter a diversity of prey. They also exhibit behavioural and physiological mechanisms for obtaining a more balanced food intake 
TABLE 3. Studies on the quality of aphids as food for generalist predators: mixed diets.

\begin{tabular}{|c|c|c|c|c|c|}
\hline Predator species & Aphid species & Comparison prey & Parameter & Effect of aphid addition & Reference \\
\hline \multicolumn{6}{|l|}{$\begin{array}{l}\text { ARANEAE } \\
\text { Linyphiidae }\end{array}$} \\
\hline \multirow[t]{4}{*}{ Erigone atra } & R. padi & D. melanogaster & $\begin{array}{l}\text { No. eggsacs/female } \\
\text { No. eggs/female } \\
\text { No. young/female }\end{array}$ & $\begin{array}{l}17 \% \text { increase } \\
26 \% \text { increase } \\
43 \% \text { increase }\end{array}$ & Toft, 1995, 1996 \\
\hline & $\begin{array}{l}\text { R. padi } \\
\text { S. avenae } \\
\text { M. dirhodum }\end{array}$ & D. melanogaster & No. eggsacs/female & $\begin{array}{l}\text { N.s. } \\
20 \% \text { decrease } \\
\text { N.s. }\end{array}$ & Bilde \& Toft, 2001 \\
\hline & $\begin{array}{l}\text { R. padi } \\
\text { S. avenae } \\
\text { M. dirhodum }\end{array}$ & D. melanogaster & No. eggs/female & $\begin{array}{l}\text { N.s. } \\
47 \% \text { decrease } \\
\text { N.s. }\end{array}$ & \\
\hline & $\begin{array}{l}\text { R. padi } \\
\text { S. avenae } \\
\text { M. dirhodum }\end{array}$ & D. melanogaster & No. young/female & $\begin{array}{l}\text { N.s. } \\
35 \% \text { decrease } \\
\text { N.s. }\end{array}$ & \\
\hline Lycosidae & & & & & \\
\hline $\begin{array}{l}\text { Pardosa prativaga juv. } \\
\text { OPILIONES } \\
\text { Phalangiidae }\end{array}$ & R. padi & D. melanogaster & Growth & N.s. & Toft, unpubl. \\
\hline Oligolophus tridens & $\begin{array}{l}\text { R. padi }+ \\
\text { S. avenae }\end{array}$ & D. melanogaster & $\begin{array}{l}\text { Growth } \\
\text { Survival }\end{array}$ & $\begin{array}{l}\text { N.s. } \\
\text { N.s. }\end{array}$ & Hvam \& Toft, in press \\
\hline \multicolumn{6}{|l|}{$\begin{array}{l}\text { COLEOPTERA } \\
\text { Carabidae }\end{array}$} \\
\hline Agonum dorsale & R. padi & D. melanogaster & Fecundity & $93 \%$ increase & Bilde \& Toft, 1994 \\
\hline Amara similata & R. padi & Pоа аппиа seeds & $\begin{array}{l}\text { Fecundity rate (no. } \\
\text { eggs/day) }\end{array}$ & N.s. & Jørgensen \& Toft, 1997a \\
\hline Bembidion lampros & R. padi & Catfood & $\begin{array}{l}\text { Egg volume } \\
\text { Fecundity rate }\end{array}$ & $\begin{array}{l}5.9 \% \text { increase } \\
56 \% \text { increase }\end{array}$ & Wallin et al., 1992 \\
\hline Harpalus rufipes larvae & R. padi & Poа аппиа seeds & Survival (days) & N.s. & Jørgensen \& Toft, 1997b \\
\hline Poecilus cupreus & R. padi & Catfood & $\begin{array}{l}\text { Egg volume } \\
\text { Fecundity rate }\end{array}$ & $\begin{array}{l}\text { N.s. } \\
\text { N.s. }\end{array}$ & Wallin et al., 1992 \\
\hline Pterostichus melanarius & R. padi & Catfood & $\begin{array}{l}\text { Egg volume } \\
\text { Fecundity rate }\end{array}$ & $\begin{array}{l}\text { N.s. } \\
\text { N.s. }\end{array}$ & Wallin et al., 1992 \\
\hline \multicolumn{6}{|l|}{ AVES } \\
\hline \multirow[t]{2}{*}{ Perdix perdix chicks } & R. padi & Locusta migratoria & $\begin{array}{l}\text { Juvenile growth } \\
\text { Food utilization (g } \\
\text { growth } / g \text { food) }\end{array}$ & $\begin{array}{l}31 \% \text { increase } \\
26 \% \text { increase }\end{array}$ & Borg \& Toft, 1999, see also 2000 \\
\hline & & & Flight feather length & $16 \%$ increase & \\
\hline
\end{tabular}

than expected from the contents of their prey (Mayntz et al., 2005) and are thus expected to have a relatively good nutrient balance, similar to those given enriched flies in the laboratory. The above results therefore point at the possibility that under field conditions negative effects of aphids should be more predominant than positive ones. This remains to be documented, however.

\section{OPTIMAL DIETARY MIXING}

When a prey like aphids both have potentially fitness increasing and fitness decreasing constituents, the ability of optimal self-selection of prey (Waldbauer \& Freedman, 1991) may secure the maximal benefit to the predator. While the development of an aversion against aphids in some generalists (Toft, 1997) may be interpreted as a mechanism to prevent fitness reductions from excessive aphid consumption, it is only an indirect indication of optimal prey selection. The question whether the generalist predators of cereal fields are able to select a mixed diet of aphids and other (high-quality) prey in optimal proportions has so far been addressed only in experiments with partridge chicks (Borg \& Toft, 1999, 2000). Diets with fixed composition of aphids (R. padi) and chopped grasshopper were produced, so that the chicks were unable to select one or the other part of the diet. The treatment foods contained $0,15,30$ and $45 \%$ aphid. In an additional treatment the aphids and chopped grasshoppers were served separately for the chicks to chose. The resulting growth curves confirmed the prediction of a maximal growth rate in the self-selection treatment. Over the 5 days of the experiment the proportion of aphids selected by the chicks was $7 \%$. The benefit of including a small amount of aphids in the diet was revealed by a significantly lower growth rate in the pure grasshopper treatment. With higher aphid proportions in the diet chick growth was seriously reduced, supporting the idea of a toxic component in the aphids.

\section{PHYSIOLOGICAL EFFECTS OF APHID CONSUMPTION ON GENERALIST PREDATORS}

Evidence that the low food quality of aphids to generalist predators is most likely due to presence of toxins or deterrents rather than to inadequate nutrient composition has already been discussed: aphid consumption is independent of hunger level; reduced predator fitness is observed when aphids are added to a high-quality diet (fruit fly or grasshopper). Exactly how cereal aphids influence the physiology of the predators is still an open question. Toft \& Nielsen (1997) found a doubling of the 
basal metabolic rate in wolf spiders kept on aphids and other low-quality prey compared to high-quality prey (Table 2). They hypothesized that this might be due to activation of detoxification enzymes, but measurements of a few detoxification systems did not confirm this (Nielsen \& Toft, 2002). However, other detoxification systems may have been involved. Similar measurements on pupae of the polyphagous aphidophage Coccinella septempunctata showed that animals raised on $R$. padi had reduced activity of glutathione S-transferase, but increased activity of a glutathione peroxidase compared to individuals raised on high-quality $M$. dirhodum (Nielsen et al., 2000). Increased activity of glutathione peroxidase may indicate some degree of oxidative stress, which may explain why $R$. padi is lower food quality than $M$. dirhodum not only to generalist predators but also to ladybird beetles (Hauge et al., 1998). However, the nature of the postulated cereal aphid toxicity is unknown, but may include digestive inhibitors [as suggested by Fisker $\&$ Toft (2004) for a toxic Collembola] as well as metabolic toxins. A suggestion that aphids contain higher amounts of undigestible, possibly plant-derived substances than other prey would be included in the toxicity concept as it is used here, if low digestibility is the cause of the low food value.

A pure diet of a cereal aphid retards development of a generalist predator. However, predators demonstrate the ability to recover from a developmental stand-still when given high-quality prey following a "stress" period of aphid feeding. Thus, wolf spiders compensated seemingly completely after just a few weeks (Jespersen \& Toft, 2003), i.e. they matured no later than individuals not inhibited by aphid feeding. In the linyphiid spider Tenuiphantes tenuis the duration of the fourth (and final juvenile) instar was greatly reduced in spiders that had the first instar prolonged by aphid feeding (Beck \& Toft, 2000).

Maternal effects of aphid feeding by females on their young may also be due to toxins from the aphids. Kyneb $\&$ Toft (in prep.) found substantial effects of maternal $R$. padi and $S$. avenae diets on eggs and larvae of the rove beetle Tachyporus hypnorum. The responses to the two aphid species differed, indicating differences in the constituents producing the effects. One of the species ( $S$. avenae) also produced a reduced hatching success of F2 eggs, demonstrating grandmaternal effects.

It is interesting to note that the quality ranking of the three cereal aphids found in the wolf spider is repeated in the aphidophagous Coccinella septempunctata, although for this species all are essential prey. Thus, M. dirhodum is of the highest quality to the ladybird larvae, $R$. padi is of lowest quality, and $S$. avenae is intermediate (Hauge et al., 1998). This may indicate that there are two systems of chemical defence in the aphids, one that makes them unavailable to generalist predators but that the aphidophages have adapted to cope with; and one that modifies the relative value of the aphid species to a lesser degree and which is effective against all predators. However, some predator-specific effects are also involved, thus $P$. prativaga and $C$. septempunctata rank the three cereal aphids similarly, but different from E. atra and T. hypnorum.

\section{GENETIC VARIATION IN TOLERANCE TO APHIDS}

In growth experiments with spiders given aphid diets there is often a substantial variation between individuals in their survival and rate of growth. Some individuals die as first instars, others moult once and actually put on some weight before dying. The hypothesis that this variation might be due to genetic variation in tolerance to aphids has been tested by an artificial selection experiment with the linyphiid spider Tenuiphantes tenuis (Beck $\&$ Toft, 2000). Hatchlings of the aphid-selection treatment were fed only $R$. padi during their first instar. Those that survived and moulted into the second instar were raised to maturity on high-quality prey (Collembola) and allowed to breed. After just one generation of selection a significant improvement in survival was revealed in the selected line compared to individuals of the control line. However, animals selected for aphid tolerance in one generation also showed a reduced survival on the highquality control food, indicating the existence of a physiological trade-off between tolerance to aphids and the ability to utilise other kinds of food.

These results lead to the yet untested prediction that a species' tolerance to aphids should be higher in areas of frequent aphid outbreaks. It is unknown however to what level of tolerance selection may take these spiders, and whether selection also may lead to a higher ability to benefit from aphid consumption.

\section{BEHAVIOURAL MECHANISMS BEHIND REDUCED APHID CONSUMPTION}

When a predator eats less of a prey than indicated by its food demand, it must be due to a rejection response not induced by satiation. Toft (1997) found that juveniles of the wolf spider Pardosa prativaga accepted cereal aphids initially, but after some experience rejected them subsequently. This indicates that they developed a learned aversion against the aphids after having captured and eaten some within a short period. The ease with which the aversion arose depended on the species of aphid tested. Thus, the animals started to reject aphids after consumption of $2.6 \mathrm{R}$. padi, $3.0 \mathrm{~S}$. avenae, or $3.5 \mathrm{M}$. dirhodum. The number of aphids needed to induce the aversion was related to their relative quality as food for the spider and independent of the size of the aphid. Thus, the more palatable the aphid, the more were needed. The aversion was temporary, however, a few hours up to one day, then another aphid was accepted. The persistence of the aversion was also related to relative food value: it disappeared earlier when induced by an aphid of lower quality, i.e. an $R$. padi-aversion faded before an $M$. dirhodum-aversion. A new aversion can be induced after consumption of additional aphids. The low number of aphids needed to induce the aversion, and the short time to forget it, make repeated cycles of induction and subsequent loss of the learned aversion a likely mechanism to explain the low 
aphid consumption rate of the generalist predators. Repeated induction may reinforce the aversion which then lasts for longer periods of time (S. Toft, unpubl.).

\section{PREY SELECTION IN GENERALIST VS. SPECIALIST PREDATORS}

Specialist predators are generally thought to have specific search images and show little predatory behaviour towards other "prey" than those showing these specific cues (cf. Bernays, 2001). In the case of aphid specialists these cues may be both visual and chemical, probably with an emphasis on the latter (Hodek \& Honěk, 1996). Thus, the cuticular waxes of aphids seem to be important for prey recognition in coccinellids (review in Dixon, 2000). A search image secures that they positively select their specialised prey which are of high nutritional quality to them and ignore other non-prey animals. Experience should play a relatively minor role in prey selection, though learning may be involved in forming a specific search image for particular prey within the limited optimal set (Jackson \& Li, 2004).

Generalist predators on the other hand, respond with a predatory attack to all potential prey of a proper size revealing some very simple, generalised cues, e.g. movement. They attack a wide range of potential prey, independently of their food quality, and have to learn which prey are high-quality and which are low-quality. Also, they may need to capture a prey in order to be able to evaluate it properly. For example, Toft \& Wise (1999b) found that, after having developed an aversion to lowquality sciarid midges, a wolf spider might continue to capture them, only to release them (often alive) after a brief check.

Thus, specialists are selective before prey capture and change preference only to a limited extent, whereas generalists are initially unselective, but become increasingly selective as a result of experience. Acquiring and loosing an aversion may be a mechanism behind self-selection of prey types, as it keeps the consumption rate of the potentially toxic prey at the optimal level where the predator gets the maximal benefit.

\section{AGGREGATIVE RESPONSE TO APHIDS?}

In the above a picture has been sketched based solely on evidence from laboratory studies. What evidence exist from the field that generalist predators respond to aphids as low-quality prey? Some studies have found that aphids formed a high proportion of prey collected from spiders' webs (Nentwig, 1980, 1982), from which it has been concluded that aphids are also a main prey of the spider. Since we have so far no evidence of any aphid being high-quality food for any spider, we should await more detailed analyses before accepting such claims. Other studies have noted a negative selectivity of spiders for aphids (e.g. Nyffeler et al., 1987). However, selectivity indices based on field sampling are influenced by a host of biological factors, irrespective of prey nutritional quality. Most studies of spider prey in the field do not distinguish between what prey is captured and what is consumed. And even this distinction may not identify low-quality prey unless these are captured frequently, as multiple captures may be needed to induce a feeding aversion (see above). One study that most directly demonstrates rejection of aphids in cereal fields is that of Alderweireldt (1994). The most abundant prey group were aphids, isotomid collembola, sciarid midges and sternorrhynch Hemiptera. Of the individuals captured by several linyphiid spider species, only $41 \%$ of aphids were consumed against 91, 94, and $81 \%$, respectively, of the other groups, indicating that spiders in the field also develop an aversion against aphids when they are captured in abundance.

Field experimental studies, e.g. by Bryan \& Wratten (1984) seemingly demonstrated an aggregative numerical response to aphid concentrations in some carabid beetles and in Tachyporus. However, such responses can only be expected in predators, which have both a high consumption capacity for aphids and which can utilize aphids to a considerable extent. Even predators, which derive a positive benefit from aphid predation but get this benefit with a low consumption rate, would not be expected to aggregate, because they might meet their aphid consumption limit without aggregating.

An hypothesis that also accounts for the empirical findings of Bryan \& Wratten (1984) is that large aphid colonies produce large amounts of honeydew, which would attract large numbers of Diptera and possibly other kinds of potential high-quality prey and therefore be attractive for generalist predators. Honeydew might also promote fungal growth and thus be attractive to fungivores (Tachyporus is both a predator and a fungivore). This alternative hypothesis was tested in a field experiment in which aphids were added to some plots and honey was sprayed to others (Monsrud \& Toft, 1998). The results confirmed that several groups of potential prey for generalist predators (in particular Diptera) were more attracted to the honey-sprayed plots than to the aphid-addition plots, and so were carabid beetles. The only predatory groups which were more attracted to the aphids than to honey, were Tachyporus and predatory beetle larvae. These are exactly the groups for which we have laboratory evidence that aphids are of sufficient quality to sustain the full development (Kyneb \& Toft, 2004; Sørensen, 1996). Thus, aggregation of carabid beetles around aphid colonies does not conflict with the concept that aphids are low-quality food, and it is not an indication that aphids are palatable to them, or that they consume aphids in high amounts.

Harwood et al. $(2003,2004)$ demonstrated that linyphiid spiders in winter wheat fields select web sites with significantly higher prey availability than average for the field. Linyphiine but not erigonine spiders selected web sites with significantly higher than average availability of aphids (Harwood et al., 2004). However, nearly all prey groups (including Collembola, Diptera) and other predators (e.g. spiders other than the web owners) were more abundant at focal web sites, so no specific attraction to aphids can be postulated. In agreement with the above 
suggestion the results show, at the scale of a spider web (i.e. a few $\mathrm{cm}$ ), a positive association between aphids and alternative prey for generalist predators, and also between alternative prey and the generalist predators themselves.

\section{CAN PREDATORS WITH LOW APHID TOLERANCE CONTRIBUTE TO THE LIMITATION OF APHID POPULATION INCREASE?}

From the results referred to above, the question arises: Does the low food quality of aphids imply that biocontrol by means of generalist predators is impossible?

We have approached this question by means of a simulation model (Axelsen \& Toft, unpubl.) based on the metabolic pool concept (Gutierrez, 1996) and incorporating the low aphid feeding capacity of the predators. The model described the growth of an aphid population under predation from a single predator population. The aphid was given biological characteristics of Rhopalosiphum padi, and the predator had characteristics of the linyphiid spider Erigone atra, a species with a low aphid consumption capacity. We considered the standard situation of a cereal field which early in spring is inhabited by a low population of generalist predators, and simulated the growth of both the aphid and the predator populations. The predators started reproduction early in spring as soon as the temperature allowed it, while the aphids were assumed to immigrate on 1 June, which is a typical arrival time in Denmark.

The simulations were run with two different predators, which had exactly the same characteristics, except that one had a low consumption capacity for aphids, whereas the other was able to satisfy its food demand by eating aphids and could utilise aphids for growth and reproduction. The simulations also included alternative prey (i.e. high-quality prey on which the predators could grow and reproduce). Thus, we simulated to what extent the aphidlimited and the aphid-unlimited predator were able to reduce the aphid population depending on the availability of alternative prey. Furthermore, we ran all these simulations under three different rates of aphid immigration: 1, 10 or 100 aphids per $\mathrm{m}^{2}$.

The results indicate that the low food quality of the aphids influences the predators' potential impact on the aphid population under some, but not all circumstances. The effect depends both on the aphid immigration rate and on the availability of alternative prey. At very low aphid immigration rate, the difference between limited and unlimited predators is very small. Even the aphidlimited predator can reduce the aphid population to approximately the same low level as the aphid-unlimited predator (ca. $2 \%$ of the aphid population not influenced by predation). At intermediate aphid immigration rate, the result indicates a substantial difference except at a narrow interval of alternative prey availability, where the limited predator was nearly as efficient as the unlimited predator. Finally, at very high aphid immigration rate, aphid limitation makes a huge difference. In this case the limited predator is only a little better than no predator.

The mechanisms behind these results are as follows: If there is no alternative prey available, the predators are unable to reproduce and die out before the aphids immigrate. (In the field these predators would probably have dispersed, which would give the same result.) Increasing the availability of alternative prey means an increasing reproduction of the predators, and thus a larger predator population will be present in the field at the time the aphids immigrate. Therefore, we initially get a reduced aphid peak the more alternative prey is available. However, with further increase in alternative prey, predator reproduction reaches its maximum, and the aphid consumption of each individual will decrease. Predators in agricultural fields may be extremely food limited (Bilde $\&$ Toft, 1998). Therefore, at low availability of alternative prey the predators will encounter relatively many aphids before they have satisfied their food demand, if they ever reach that level. This means that they will eat all aphids encountered, up to their aphid consumption capacity. With a high availability of alternative prey, the predators will satisfy their food demand before reaching the aphid consumption limit. Therefore, the predators will reduce the aphid peak less at very high availability of alternative prey.

Data from the field are now available that partly confirm the postulated interaction between abundance of alternative prey and predation on aphids. Harwood et al. (2004) found that both the amount and the concentration of aphid proteins in linyphiid spiders increased dramatically in a situation where the numbers of collembola dropped even though the aphid population remained constant.

\section{PERSPECTIVES}

The results presented here give us a plausible explanation for the very different roles of generalist and aphid specialist predators in the biocontrol of aphids in cereal fields. Basically, it is the aphids' poor quality as food for generalists that limits aphid consumption, induced by a specific feeding aversion. Aphids are a minor food source that is accepted if encountered at frequencies too low to induce an aversion. They are taken because generalist predators have to test a prey to learn if it is good or bad. Provided alternative prey is available to boost the predator population, the consumption of a predator population may be sufficient to suppress aphid populations. This effect may be further enhanced if one considers the full assemblage of often more than a hundred species of generalist predators, which together will not only reach higher predator densities than any single species, but due to microhabitat segregation between species will also leave fewer refuges for aphids (Sunderland et al., 1997).

Proponents of the use of generalist predators in biological (conservation) control of insect pests have argued against the necessity of prey specialization as a prerequisite for an effective biocontrol agent. However, theoretical considerations (Provencher \& Riechert, 1995; Chang \& Kareiva, 1999; Riechert et al., 1999) still consider that for generalist predators to be effective in biocontrol, their opportunistic feeding habits should enable them to exploit a growing pest population through an 
aggregative numerical response. This assumes that the pest is a high quality prey for the predator.

The aphid-generalist predator interactions in cereal fields do not follow the assumptions of any of these models: the pest is unpalatable to the predators that are supposed to control them, so that each predator is limited to eating a few; the pest contributes little or perhaps even negatively to predator fitness. Still, we may (sometimes) observe a significant level of biocontrol. This effect hinges on the generalist predator populations being dependent on a resource base of the detrital food web (Settle et al., 1996; Harwood et al., 2004) rather than being coupled to aphid population dynamics. This enhances the prospects for biocontrol of aphids, because the detrital food web can be manipulated by techniques that are already standard agricultural practices, e.g. by manuring or mulching (Sunderland \& Samu, 2000).

The challenge for ecologists now is to quantify the conditions under which generalist predators may have maximal effectiveness and those under which they are ineffective. One remaining question is whether it is possible to increase predator density and diversity to such levels that, even under conditions favouring a high aphid immigration rate to the cereal fields, the generalist predator assemblage may prevent an outbreak. In connection with this it also remains to be established if the levels of alternative prey can be so high as to pass the optimum level for biocontrol.

The situation of a pest being low quality food for generalist predators, is not unique to cereal aphids. Data are available from other systems with similar relationships between the pests and their associated generalist predators. Thus, Sigsgaard et al. (2001a, b) found that planthoppers of East-asian rice crops were also lowquality food for the dominant spiders species of these crops. Planthopper control by spiders in rice paddy fields has often been cited as the most effective and best documented instances of pest control by a natural spider fauna (cf. Riechert \& Lockley, 1984). A third example may be that of control of field slugs Deroceras reticulatum by carabid beetles in the UK. Bohan et al. (2000) found "spatial density dependence" between the slugs and the carabid beetle Pterostichus melanarius, resulting in significant local reductions in slug densities. However, laboratory tests have demonstrated low food quality of these slugs for $P$. melanarius and other carabid species (Mair \& Port, 2001a, b; Fawki et al., 2005).

It can be hypothesized that many invertebrates due to chemical defences escape control by generalist predators, because a low feeding capacity requires a high predator/prey ratio for control to be effective. It may even be expected that several insects owe their pest status to chemical defences that make them unpalatable to generalist predators and therefore prevent numerical responses. The often low predator densities in conventional cropping systems will therefore result in quite low "accidental" predation simply due to a low predator/prey ratio. However, exactly because of their generalistic feeding habits, and because the same types of prey seem to be nutrition- ally high-quality food for all generalist predators (cf. Toft $\&$ Bilde, 2002), the same management measures may turn out to be effective over a wide range of situations, i.e. independent of which species form the local pest complex and of the local fauna of generalist predators.

ACKNOWLEDGEMENTS. I am indebted to several colleagues and students that have contributed to our understanding of aphid-generalist predator relationships presented here: J.A. Axelsen, J.B. Beck, T. Bilde, C. Borg, S. Fawki, E. Nølke Fisker, M.S. Hauge, A. Hvam, L.B. Jespersen, H. Bracht Jørgensen, A. Kyneb, M. Madsen, B.M. Marcussen, D. Mayntz, C. Monsrud, F.F. Nielsen, S. Achim Nielsen, J. Offenberg, L. Sigsgaard, T. Nørgaard, F. Sørensen, S. Terkildsen and D.H. Wise. The original work referred to was funded by grants from the Danish Environmental Research Program, the Danish Research Centre for Organic Farming, the National Science Research Council, and the Carlsberg Foundation.

\section{REFERENCES}

AlderweireldT M. 1994: Prey selectiom and prey capture strategies of linyphiid spiders in high-input agricultural fields. Bull. Brit. Arachnol. Soc. 9: 300-308.

Beск J.B. \& Tоғт S. 2000: Artificial selection for aphid tolerance in the polyphagous predator, Lepthyphantes tenuis (Araneae: Linyphiidae). J. Appl. Ecol. 37: 547-556.

BERNAYS E.A. 2001: Neural limitations in phytophagous insects: implications for diet breadth and evolution of host afiliation. Annu. Rev. Entomol. 46: 703-727.

BILDE T. \& TofT S. 1994: Prey preference and egg production of the carabid beetle Agonum dorsale. Entomol. Exp. Appl. 73: 151-156.

BILDE T. \& TofT S. 1997a: Limited predation capacity by generalist arthropod predators on the cereal aphid, Rhopalosiphum padi. Org. Agric. Hort. 20: 143-150.

BILDE T. \& TOFT S. 1997b: Consumption by carabid beetles of three cereal aphid species relative to other prey types. Entomophaga 42: 21-32.

BILDE T. \& ToFT S. 1998: Quantifying food limitation of arthropod predators in the field. Oecologia 115: 54-58.

Bilde T. \& Toft S. 1999: Prey consumption and fecundity of the carabid beetle Calathus melanocephalus on diets of three ceral aphids: high consumption rates of low-quality prey. Pedobiologia 43: 422-429.

BiLde T. \& TofT S. 2000: Evaluation of prey for the spider Dicymbium brevisetosum Locket (Araneae: Linyphiidae) in single-species and mixed-species diets. Ekológia (Bratislava) (Suppl. 3) 19: 9-18.

Bohan D.A., Bohan A.C., Glen D.M., Symondson E.O.C., Wiltshire C.W. \& Hughes L. 2000: Spatial dynamics of predation by carabid beetles on slugs. J. Anim. Ecol. 69: 367-379.

Borg C. \& Toft S. 1999: Value of the aphid Rhopalosiphum padi as food for partridge Perdix perdix chicks. Wildl. Biol. 5: $55-58$.

Borg C. \& Toft S. 2000: Importance of insect prey quality for grey partridge chicks (Perdix perdix): a self-selection experiment. J. Appl. Ecol. 37: 557-563.

BRYAN K.M. \& WRATten S.D. 1984: The responses of polyphagous predators to prey spatial heterogeneity: aggregation by carabid and staphylinid beetles to their cereal aphid prey. Ecol. Entomol. 9: 251-259.

Chang G.C. \& Kareiva P. 1999: The case for indigenous generalist predators in biological control. In Hawkins B.A. \& Cornell H.V. (eds): Theoretical Approaches to Biological 
Control. Cambridge University Press, Cambridge, pp. $103-115$

Dixon A.F.G. 2000: Insect Predator-Prey Dynamics. Ladybird Beetles \& Biological Control. Cambridge University Press, Cambridge, $257 \mathrm{pp}$.

Edwards C.A., Sunderland K.D. \& George K.S. 1979: Studies on polyphagous predators of cereal aphids. J. Appl. Ecol. 16: 811-823.

Evans E.W. \& Gunther D.I. 2005: The link between food and reproduction in aphidophagous predators: a case study with Harmonia axyridis (Coleoptera: Coccinellidae). Eur. J. Entomol. 102: 423-430.

FAWKI S., BAK S.S. \& ToFT S. 2005: Food preferences and food value in the generalist carabid beetles Pterostichus melanarius, P. versicolor and Carabus nemoralis. In Lövei G. \& Toft S. (eds): European Carabidology 2003. Proceedings of the 11th European Carabidologist Meeting. DIAS Reports No. 114, Danish Institute of Agricultural Sciences, pp. 99-109.

FISKER E.N. \& ToFT S. 2004: Effects of chronic exposure to a toxic prey in a generalist predator. Physiol. Entomol. 29: $129-138$.

Gutierrez A.P. 1996: Applied Population Ecology. A SupplyDemand Approach. John Wiley \& Sons, New York, 320 pp.

Harwood J.D., SunderLand K.D. \& SyMONDSON W.O.C. 2001: Living where the food is: web location by linyphiid spiders in relation to prey availability in winter wheat. J. Appl. Ecol. 38: 88-99.

Harwood J.D., Sunderland K.D. \& Symondson W.O.C. 2003 Web-location by linyphiid spiders: prey-specific aggregation and foraging strategies. J. Anim. Ecol. 72: 745-756.

Harwood J.D., SunderLand K.D. \& Symondson W.O.C. 2004 Prey selection by linyphiid spiders: molecular tracking of the effects of alternative prey on rates of aphid consumption in the field. Mol. Ecol. 13: 3549-3560.

Hauge M.S., Nielsen F.H. \& Toft S. 1998: The influence of three cereal aphid species and mixed diet on larval survival, development and adult weight of Coccinella septempunctata. Entomol. Exp. Appl. 89: 319-322.

HodeK I. \& HonĚK A. 1996: Ecology of Coccinellidae. Kluwer Academic Publishers, Dordrecht, $464 \mathrm{pp}$.

Hvam A. \& Toft S. 2005: Effects of prey quality on the life history of a harvestman. J. Arachnol. (in press).

JACKSON R.R. \& Li D. 2004: One-encounter search-image formation by araneophagic spiders. Anim. Cogn. 7: 247-254.

JørGENSEN H.B. \& TOFT S. 1997a: Role of granivory and insectivory in the life cycle of the carabid beetle Amara similata. Ecol. Entomol. 22: 7-15.

JøRGENSEN H.B. \& TofT S. 1997b: Food preference, diet dependent fecundity and larval development in Harpalus rufipes (Coleoptera: Carabidae). Pedobiologia 41: 307-315.

Kyneb A. \& Toft S. 2004: Quality of two aphid species (Rhopalosiphum padi and Sitobion avenae) as food for the staphylinid generalist predator Tachyporus hypnorum. J. Appl. Entomol. 128: 158-163.

LufF M.L. 2005: Biology and ecology of immature stages of ground beetles (Carabidae). In Lövei G.L. \& Toft S. (eds): European Carabidology 2003. Proceedings of the $11^{\text {th }}$ European Carabidologist Meeting. DIAS Report No. 114. Danish Institute of Agricultural Sciences, pp. 183-208.

Madsen M., Terkildsen S. \& Toft S. 2004: Microcosm studies on control of aphids by generalist arthropod predators: effects of alternative prey. BioControl 49: 483-504.

Mair J. \& Port G.R. 2001a: Predation by the carabid beetles Pterostichus madidus and Nebria brevicollis is affected by size and condition of the prey Deroceras reticulatum. Agr. Forest Entomol. 3: 99-106.

Mair J. \& Port G.R. 2001b: Predation on the slug Deroceras reticulatum by the carabid beetles Pterostichus madidus and Nebria brevicollis in the presence of alternative prey. Agr. Forest Entomol. 3: 169-174.

Mayntz D., Raubenheimer D., SAlomon M., Toft S. \& Simpson S.J. 2005: Nutrient specific foraging in invertebrate predators. Science 307: 111-113.

MAYNTZ D. \& ToFT S. 2001: Nutrient composition of the prey's diet affects growth and survivorship of a generalist predator. Oecologia 127: 207-213.

MonsRud C. \& ToFT S. 1999: The aggregative numerical response of polyphagous predators to aphids in cereal fields: attraction to what? Ann. Appl. Biol. 134: 265-270.

Nentwig W. 1980: The selective prey of linyphiid-like spiders and their space webs. Oecologia 45: 236-243.

Nentwig W. 1982: Zur Biologie der Schilfsackspinne Clubiona phragmitis. Entomol. Abhandl. Staatl. Mus. Tierk. Dresden 45: 183-193.

Nielsen S.A. \& Toft S. 2002: Responses of a detoxification enzyme to diet quality in a wolf spider, Pardosa prativaga. In Toft S. \& Scharff N. (eds): European Arachnology 2000. Aarhus University Press, Aarhus, pp. 65-70.

Nielsen S.A., Hauge M., Nielsen F.H. \& Toft S. 2000: Activities of glutathione S-transferase and glutathione peroxidases related to diet quality in an aphid predator, the seven-spot ladybird, Coccinella septempunctata L. (Coleoptera: Coccinellidae). ATLA 28: 445-449.

NøRGaARD T. 1998: Food Preference and Food Searching by the German Cockroach Blatella germanica (L.). Three Behavioural Experiments. Masters Thesis. Department of Zoology, Aarhus University, Århus.

Oelbermann K. \& Scheu S. 2002: Effects of prey type and mixed diets on survival, growth and development of a generalist predator, Pardosa lugubris (Araneae: Lycosidae). Basic Appl. Ecol. 3: 285-291.

OfFENBERG J. 1999: Aspects of Ant-Aphid Associations and their Evolutionary Ecology. Masters Thesis. Department of Zoology, Aarhus University, Århus.

Provencher L. \& RIECHERT S.E. 1995: Theoretical comparisons of individual success between phenotypically pure and mixed generalist predator populations. Ecol. Model. 82: 175-191.

RIECHERT S.E. \& LOCKLEY T. 1984: Spiders as biological control agents. Annu. Rev. Entomol. 29: 299-320.

Riechert S.E., Provencher L. \& Lawrence K. 1999: The potential of spiders to exhibit stable equilibrium point control of pests: tests of two criteria. Ecol. Appl. 9: 365-377.

Schmidt M.H., Laur A., Purtauf T., Thies C., Schaefer M. \& TsChARNTKe T. 2003: Relative importance of predators and parasitoids for cereal aphid control. Proc. R. Soc. Lond. (B) 270: 1905-1909.

Settle W.H., Ariawan H., Astuti E.T., Cahyana W., Hakima A.L., Hindayana D., Lestari A.S. \& Pajarningsih S. 1996: Managing tropican rice pests through conservation of generalist natural enemies and alternative prey. Ecology 77: 1975-1988.

Sigsgaard L., Toft S. \& Villareal S. 2001a: Diet-dependent survival, development and fecundity of the spider Atypena formosana (Oi) (Araneae: Linyphiidae) - implications for biological control in rice. Biocontr. Sci. Techn. 11: 233-244.

Sigsgaard L., Toft S. \& Villareal S. 2001b: Diet-dependent facundity of the spiders Atypena formosana and Pardosa pseudoannulata, predators in irrigated rice. Agr. Forest Entomol. 3: 285-295. 
Sunderland K. \& SAmu F. 2000: Effects of agricultural diversification on the abundance, distribution, and pest control potential of spiders: a review. Entomol. Exp. Appl. 95: 1-13.

Sunderland K.D., Topping C.J., Ellis S., Long S., Van de LaAK S. \& ElSE M. 1996: Reproduction and survival of linyphiid spiders, with special reference to Lepthyphantes tenuis (Blackwall). In Booij C.J.H. \& den Nijs L.J.M.F. (eds): Arthropod Natural Enemies in Arable Land. II. Survival, Reproduction and Enhancement. Aarhus University Press, Århus, Acta Jutlandica 71-2: 81-95.

Sunderland K.D., Axelsen J.A., Dromph K., Freier B., Hemptinne J.-L., Holst N.H., Mols P.J.M., Petersen M.K., Powell W., Ruggle P., Triltsch H. \& Winder L. 1997: Pest control by a community of natural enemies. In Powell W. (ed.): Arthropod Natural Enemies in Arable Land. III. The Individual, the Population and the Community. Aarhus University Press, Århus, Acta Jutlandica 72-2: 271-326.

Symondson W.O.C., Sunderland K.D. \& Greenstone M.H. 2002: Can generalist predators be effective biocontrol agents? Annu. Rev. Entomol. 47: 561-594.

Sørensen O.F. 1996: The Effects of Day Length, Temperature and Prey Type on Food Consumption by Bembidion lampros and Tachyporus hypnorum. Masters Thesis. Department of Zoology, Aarhus University, Århus.

TofT S. 1995: Value of the aphid Rhopalosiphum padi as food for cereal spiders. J. Appl. Ecol. 32: 552-560.

ToFT S. 1996: Indicators of prey quality for arthropod predators. In Booij C.J.H. \& den Nijs L.J.M.F. (eds): Arthropod Natural
Enemies in Arable Land. II. Survival, Reproduction and Enhancement. Aarhus University Press, Århus, Acta Jutlandica 71-2: 107-116.

Toft S. 1997: Acquired food aversion of a wolf spider to three cereal aphids: intra- and interspecific effects. Entomophaga 42: 63-69.

Toft S. \& Bilde T. 2002: Carabid diets and food value. In Holland J.M. (ed.): The Agroecology of Carabid Beetles. Intercept, Andover, pp. 81-110.

Toft S. \& Nielsen S.A. 1997: Influence of diet quality on the respiratory metabolism of a wolf spider Pardosa prativaga. In M. Żabka (ed.): Proceedings 16th European Colloquium of Arachnology. Wyższa Szkoła Rolniczo-Pedagogiczna, Siedlce, Poland, pp. 301-307.

ToFT S. \& WisE D.H. 1999a: Growth, development and survival of a generalist predator fed single- and mixed-species diets of different quality. Oecologia 119: 191-197.

Toft S. \& Wise D.H. 1999b: Behavioral and ecophysiological responses of a generalist predator fed single- and mixedspecies diets of different quality. Oecologia 119: 198-207.

Wallin H., Chiverton P.A., Eкbom B.S. \& Borg A. 1992: Diet, fecundity and egg size in some polyphagous predatory carabid beetles. Entomol. Exp. Appl. 65: 129-140.

Winder L., Hirst D.J., Carter N., Wratten S.D. \& Sopp P.I. 1994: Estimating predation on the grain aphid Sitobion avenae by polyphagous predators. J. Appl. Ecol. 31: 1-12.

Received March 1, 2005; revised and accepted May 10, 2005 